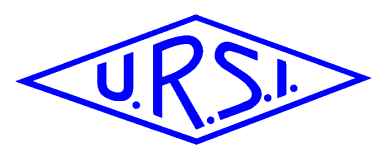

\title{
A Supervisory System for Partial Discharge Monitoring
}

\author{
B. I. Saeed(1), D. W. Upton ${ }^{(1)}$, M. F. Q. Vieira ${ }^{(2)}$, F. Torres ${ }^{(2)}$, H. Mohamed ${ }^{(1)}$, K. Mistry ${ }^{(1)}$, P. J. Mather ${ }^{(1)}$, P. Lazaridis ${ }^{(1)}$, U. \\ $\operatorname{Khan}^{(1)}$, C. Tachtatzis ${ }^{(3)}$, R. Atkinson ${ }^{(3)}$, M. Judd ${ }^{(4)}$, and I. A. Glover ${ }^{(1)}$ \\ (1) Department of Engineering and Technology, University of Huddersfield, Huddersfield HD1 3DH, UK \\ (2) Departamento de Engenharia Eletrica, Universidade Federal de Campina Grande, 58429-900 Campina Grande, PB, Brazil \\ (3) Department of Electronic and Electrical Engineering, University of Strathclyde, Glasgow G1 1XW, UK
}

(4) High Frequency Diagnostics and Engineering Ltd., Glasgow G14 0BX, UK

\begin{abstract}
Continuous measurement of partial discharge plays a vital role in monitoring the status of high voltage equipment in electricity substations. This requires a comprehensive supervisory system to detect, monitor, collect and locate partial discharge activities. Most of the sensors developed so far require a dedicated software and hardware platform to integrate them into a system. A comprehensive supervisory system is proposed and designed that integrates broadband radiometric sensors into a robust wireless network. The network is easy to install and reconfigure. Partial discharge activities are collected by the sensors and transferred to a central database where processed to provide monitoring, visualisation and localisation of the partial discharge sources.
\end{abstract}

\section{Introduction}

Partial discharge (PD) refers to a discharge that partially bridges the insulation between conductors of high-voltage (HV) equipment [1]. PD occurs in power systems insulation that is inhomogeneous in dielectric constant due, for example, to voids or cracks in solid insulation and gas bubbles or particulate contaminants in oil insulation. The occurrence of $\mathrm{PD}$ is known to be characteristic of insulation defects in HV equipment [2].

Recent research has made PD detection sensors based on various technologies possible. High frequency current transformers (HFCTs) [3], transient earth voltage (TEV) detectors [4], internal UHF antennas [5], and radiometric PD sensors [6-7] all represent practical RF sensors that are being used to monitor and locate PD occurring in cables, switchgear and transformers. Most of these sensors require a dedicated software and hardware platform to integrate them into a system so they can be efficiently utilised to monitor and locate PDs [8].

In this paper, a comprehensive supervisory system is designed to detect, monitor, and locate PD activities in electricity substation. Broadband PD radiometer sensors are designed and interfaced to a robust low power wireless system to facilitate PD data collection. A PD supervisory application is designed to monitor, locate and analyse PD data. The results show that the system is highly effective and provides a complete supervision platform for PD monitoring in substations. The remainder of this paper is organised as follows: section 2 presents an overview of the system. PD radiometer sensors are illustrated in section 3. The wireless network system is explained in section 4. Section 5 provides an overview of the PD supervisory application. Results from deploying the system in a live electricity substation are presented in section 6 . Finally, section 7 outlines some conclusions.

\section{System Overview}

Figure 1 shows the diagram of the system that comprises three main parts: PD radiometer sensors, WirelessHART network, and PD supervisory application. A collection of broadband radiometer PD sensors is deployed in a highvoltage compound to detect PD activities. The PD sensors are networked together via a mesh network of WirelessHART transceivers to facilitate continuous data collection and recording. The recorded data is then utilised by a PD supervisory application to provide continuous detection, monitoring, and location of PD activities in the HV compound. The PD supervisory application provides a complete platform to collect, monitor and visualise PD data.

\section{PD Radiometer Sensor}

The PD radiometer sensor, shown in Figure 2, comprises a radiometer antenna, an RF front-end, a signal conditioning unit and a microcontroller.

\subsection{RF-Front End}

A receiving dipole antenna provides a relatively wideband frequency, omni-directional, response of 20-1000 $\mathrm{MHz}$ with a vertical polarisation. The antenna receives radio frequency $\mathrm{PD}$ impulses emitted from defected insulations. The signal is then passed through a band-pass 


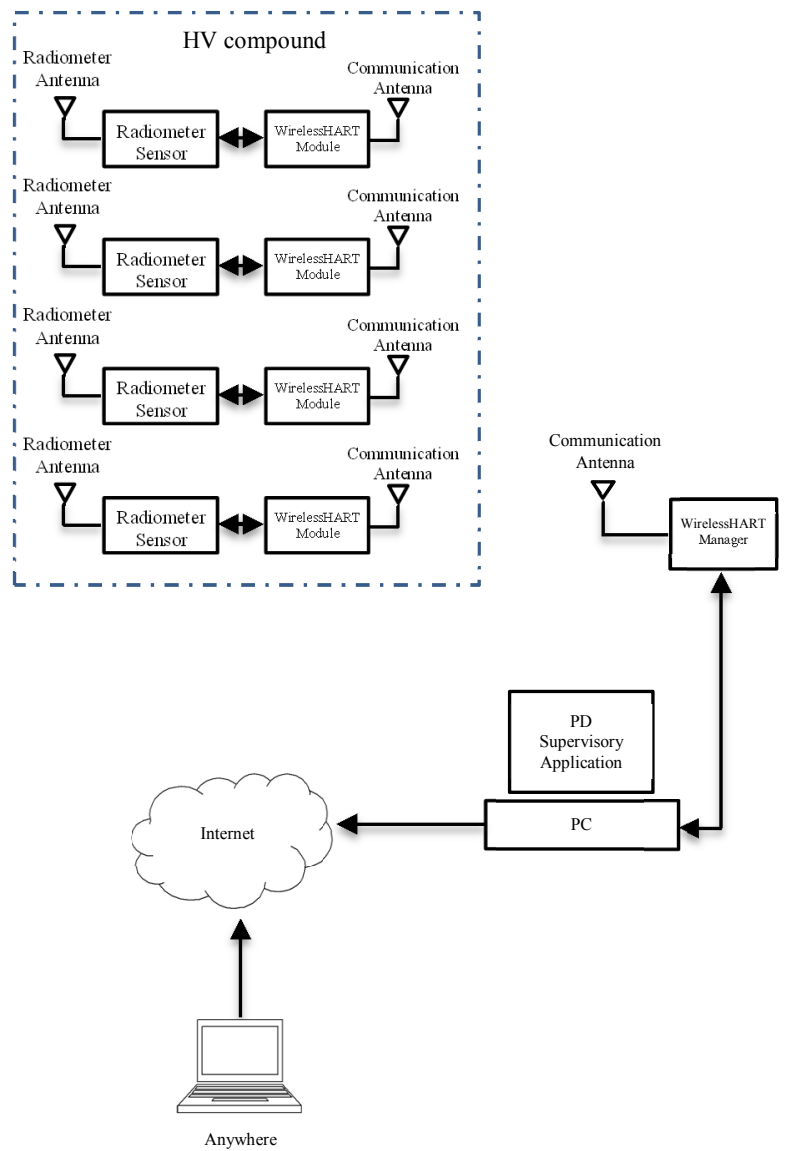

Figure 1: PD Supervisory System Diagram.

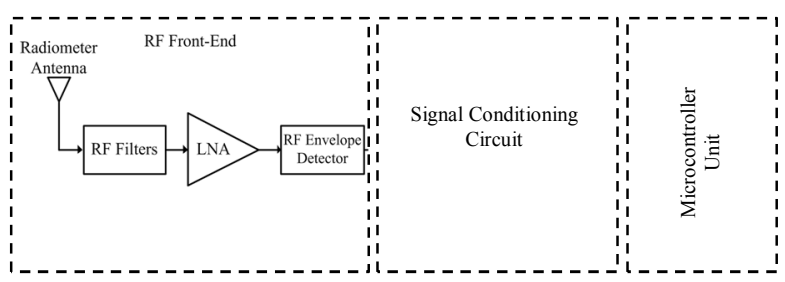

Figure 2: PD Radiometer Sensor.

roofing filter, a band-stop filter, low noise amplifier (LNA) and detector. As the frequency ranges of a variety of PDs typically reside between $50-800 \mathrm{MHz}$, with the majority of the energy below $300 \mathrm{MHz}$, the sensor measurement band was selected within this range $[9,10]$.

The roofing filter excludes all interference and noise outside the $30-320 \mathrm{MHz}$ PD measurement band. The band-stop filter removes coherent interference in the region of $70-250 \mathrm{MHz}$ (principally arising from FM radio and $\mathrm{DAB}$ transmissions). The LNA provides a fixed gain of $16.5 \mathrm{~dB}$ to increase the sensor sensitivity. Due to the filters and LNA, the RF front-end has a frequency response with two main bands of approximately 30-70 $\mathrm{MHz}$ and $250-320 \mathrm{MHz}$, with a pass-band gain between $12-14 \mathrm{~dB}$, and a noise figure in the range of 5-7 dB. This provides 20 metres of measuring range from a $\mathrm{PD}$ source.
The RF envelope detector reduces the bandwidth of the PD signal by removing the RF components, leaving the envelope.

\subsection{Signal Conditioning Circuit}

The envelope detected PD signal is applied to the signal conditioning circuit which provides further amplification before integrating it. It provides an output which is a metric of accumulated partial discharge activity. The integrator is reset to zero when its output reaches a fixed threshold which is 3 volts. A comparator is used to determine if a signal of sufficient size to be of PD interest is present and, if so, activate the integrator. This is to prevent envelope-detected noise from creating a constant rise in integrator output voltage. The comparator also counts the number of received individual PD events. Once the signal voltage drops below the threshold, integration is stopped and the output of the integrator is thus held at a constant level. The circuit provides two parameters to the microcontroller unit which are the integrator step size and the number of received PD pulses.

\subsection{Microcontroller Unit}

This unit interfaces the PD sensor circuitry to the WirelessHART module. It is designed around a PIC24EP512GU810 type microcontroller, from Microchip, that features a high speed of 70 Million Instructions Per Second (MIPS) (140 MHz), $52 \mathrm{~KB}$ of RAM and $512 \mathrm{~KB}$ of program memory. The unit also offers satisfying analogue-to-digital conversion (ADC) performance, configurable as 10-bit, 1.1 Mega samples per second $(\mathrm{MSa} / \mathrm{s})$ with up to four simultaneous channels. This ensures that fast PD pulses are adequately sampled.

The microcontroller also establishes a wireless network via the WirelessHART module and maintains the connection. To preserve energy, the microcontroller is switched to sleep mode until the data collection is due. The unit wakes up from sleep mode when it receives a signal from the PD supervisory application to collect PD data and transfer it to the system. The microcontroller monitors PD occurrences for 1 second which covers 50 periods of the main power supply. During this time, the number of PD pulses is counted, the integrator step size is sampled, and a relative time stamp of PD pulses is recorded. The average step size is then calculated and the data is transferred to the PD supervisory application via the WirelessHART modules. This process is repeated on an hourly basis.

\section{WirelessHART network}

Interfacing PD sensors with a robust wireless network is vital for providing a continuous PD monitoring system. Wireless communications technology such as WirelessHART (IEC 62591) makes a constant PD 
monitoring and scalable system possible. In addition, it provides a low-cost, flexible, and easy to install system. WirelessHART [11], based on IEEE 802.15.4, is lowpower, self-forming multi-hop, mesh-network technology. The network communicates using a Time Slotted Channel Hopping (TSCH) link layer and it is specifically designed for deployment in industrial environments where reliability and resilience are essential. In a $\mathrm{TSCH}$ network, timeslots are allocated to each node, which enables collision-free packet exchange and pertransmission channel-hopping. The $2.4 \mathrm{GHz}$ band used by WirelessHART is several times the highest frequency in the PD measurement band, has no interference with the radiometer PD sensors. The network is first created and coordinated by the WirelessHART Manager, and then the WirelessHART Modules are joined to the network to form a mesh network, thus providing a secure and reliable communication infrastructure to the PD supervisory system.

\section{PD Supervisory Application}

The PD supervisory application encompasses three main software modules: PD data collector, monitoring and visualisation module, and localization algorithm. The PD data collector interacts with the WirelessHART Manager to obtain the PD data and store it in a temporary database. The monitoring and visualisation module is built on the Supervisory System: Indusoft Web Studio Schneider/Wonderware [12], in its Academic Version. It has a built-in MySQL database system. Figure 3 shows the main elements of the system.

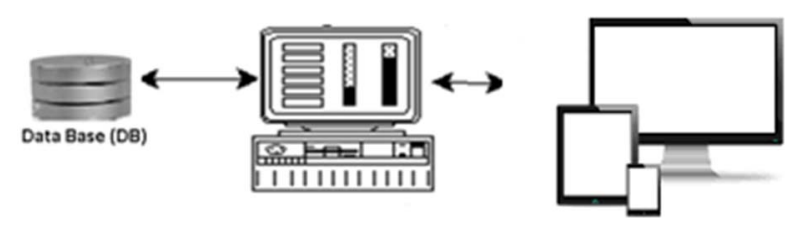

Figure 3: PD Supervisory Application.

The supervisory application offers the user a set of functions, which are illustrated in Figure 4.

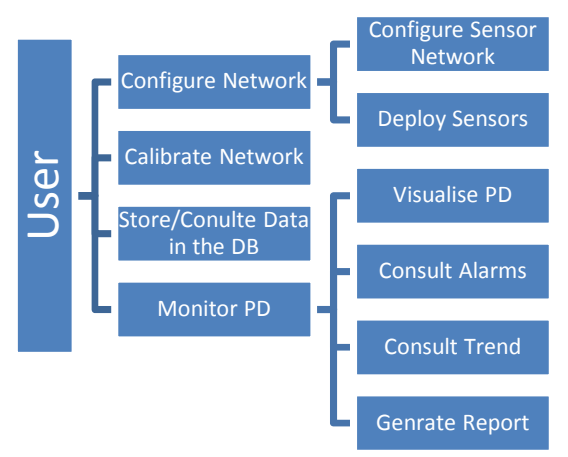

Figure 4: Supervisory Functions.
In Figure 5, each circle represents one sensor deployed on the power plant site, with the circle diameter representing the PD intensity measured by each sensor at a certain point in time. The supervisory application also enables the user to consult current and historic PD sensor data, making it possible to compare the sensor data with set points, as well as generating alarms; drawing trend diagrams; and generating periodical reports. In addition, various localisation algorithms can be integrated with the system to locate PD sources.

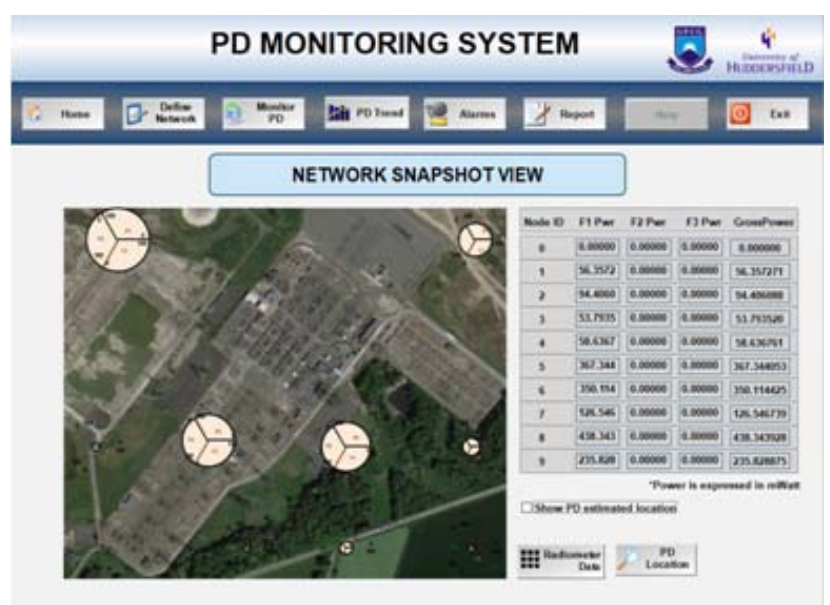

Figure 5: A Network Snapshot View of the Measured PD.

\section{Electricity Substation Deployment Tests and Results}

A network of nine radiometric PD sensors was deployed in a live $67 \mathrm{kV}$ electricity substation where transformers, circuit breakers, and bus bars were included. A grid of 16 $\mathrm{x} 16$ metres with a spacing of 8 metres between the nodes was formed. Before conducting the test, a manual PD survey was carried out to detect possible PD activities. In this survey the Doble PDS100 Partial Discharge Surveyor and handheld spectrum analyser were used where no significant PD activities were detected. In order to calibrate the system and to generate PD pulses a commercial HVPD pC (picocoulomb) pulse generator connected to Aaronia BicoLOG 20100E wideband biconical antenna was used. The device provides pulses from $1 \mathrm{pC}$ to $100 \mathrm{nC}$ with selectable pulse repetition rate $(100,120,400 \mathrm{~Hz})$. The PD emulator was set to generate $10 \mathrm{nC}$ pulses at a repetition rate of $120 \mathrm{~Hz}$. At three different positions of PD source measurements were taken. For each position the test was repeated several times. Figure 6 shows the results from one of the tests. As can be seen, number of pulses per second (PPS) and received signal power in $\mathrm{dBm}$ of each radiometric senor are indicated. The estimated location of the PD source was calculated with an error of $1.3 \mathrm{~m}$. 


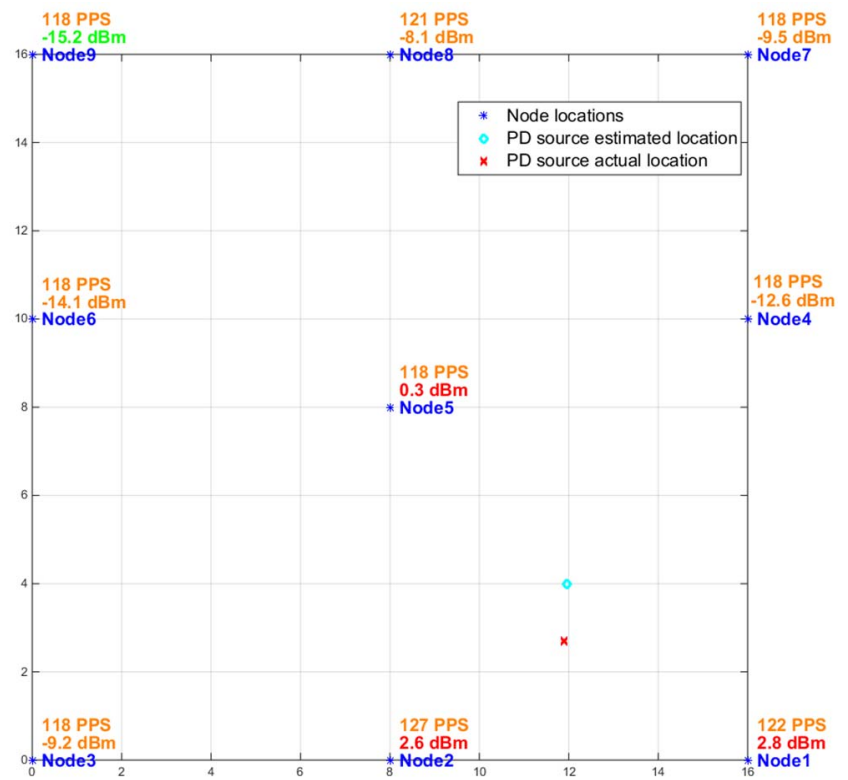

Figure 6: Estimated location of a PD source.

\section{Conclusion}

A comprehensive PD supervisory system for monitoring, visualising and locating PD has been designed, implemented and deployed in a live electricity substation. The system has been demonstrated to effectively visualise an emulated PD source. The source has been located with useful accuracy.

\section{Acknowledgements}

This work was supported by the U.K. Engineering \& Physical Sciences Research Council under Grant $\mathrm{EP} / \mathrm{J} 015873$.

\section{References}

1. IEEE Standard for High-Voltage Switchgear (Above 1000 V) Test Techniques - Partial Discharge Measurements," in IEEE Std C37.301-2009, vol., no., pp.c1-63, $\quad$ March $20 \quad 2009$ doi: 10.1109/IEEESTD.2009.4804311

2. P. H. F. Morshuis, "Degradation of solid dielectrics due to internal partial discharge: some thoughts on progress made and where to go now," in IEEE Transactions on Dielectrics and Electrical Insulation, vol. 12, no. 5, pp. 905-913, Oct. 2005. doi: 10.1109/TDEI.2005.1522185

3. X. Hu, W. H. Siew, M. D. Judd and X. Peng, "Transfer function characterization for HFCTs used in partial discharge detection," in IEEE Transactions on Dielectrics and Electrical Insulation, vol. 24, no. 2, pp. 1088-1096, April 2017. doi: 10.1109/TDEI.2017.006115

4. E. Jennings and A. Collinson, "A partial discharge monitor for the measurement of partial discharges in a high voltage plant by the transient earth voltage technique," 1993 International Conference on Partial Discharge, Canterbury, 1993, pp. 90-91. URL: http://ieeexplore.ieee.org/stamp/stamp.jsp?tp=\&arn umber $=341424 \&$ isnumber $=7987$

5. A. M. Ishak, M. T. Ishak, M. T. Jusoh, S. F. Syed Dardin and M. D. Judd, "Design and Optimization of UHF Partial Discharge Sensors Using FDTD Modeling," in IEEE Sensors Journal, vol. 17, no. 1, pp. 127-133, Jan.1, 1 2017. doi: 10.1109/JSEN.2016.2628035

6. Y. Zhang et al., "Radiometric Wireless Sensor Network Monitoring of Partial Discharge Sources in Electrical Substations," International Journal of Distributed Sensor Networks, vol. 11, Article ID 438302, 9 pages http://dx.doi.org/10.1155/2015/438302

7. D. W. Upton et al., "Wireless sensor network for radiometric detection and assessment of partial discharge in HV equipment," 2017 XXXIInd General Assembly and Scientific Symposium of the International Union of Radio Science (URSI GASS), Montreal, QC, 2017, pp. 1-4. doi: 10.23919/URSIGASS.2017.8104973

8. H. Mohamed et al., "Partial discharge detection in smart grid using software defined radio," 2017 25th Telecommunication Forum (TELFOR), Belgrade, 2017, pp. 1-4. doi: 10.1109/TELFOR.2017.8249457

9. S. Xiao, P. J. Moore and M. Judd, "Investigating the assessment of insulation integrity using radiometric partial discharge measurement," 2009 International Conference on Sustainable Power Generation and Supply, Nanjing, 2009, pp. 1-7. doi: 10.1109/SUPERGEN.2009.5347978

10. A. A. Jaber et al., "Calibration of free-space radiometric partial discharge measurements," in IEEE Transactions on Dielectrics and Electrical Insulation, vol. 24, no. 5, pp. 3004-3014, Oct. 2017. doi: 10.1109/TDEI.2017.006730

11. D. Chen, M. Nixon, S. Han, A. K. Mok and X. Zhu, "WirelessHART and IEEE 802.15.4e," 2014 IEEE International Conference on Industrial Technology (ICIT), Busan, 2014, pp. 760-765. doi: 10.1109/ICIT.2014.6895027

12. Indusoft.com. (2018). InduSoft Web Studio HMI SCADA Development Software | Wonderware by Schneider Electric. [online] Available at: http://www.indusoft.com/ [Accessed 25 Jan. 2018]. 